\title{
НЕИНВАЗИВНЫЙ ПРЕНАТАЛЬНЫЙ МОЛЕКУЛЯРНЫЙ СКРИНИНГ: ОСОБЕННОСТИ ВНЕДРЕНИЯ В КЛИНИЧЕСКУЮ ПРАКТИКУ
}

\author{
Д. О. Коростин ${ }^{1,2}$, Д. А. Плахина², В. А. Белова ${ }^{1,2}$ \\ ' Российский национальный исследовательский медицинский университет имени Н. И. Пирогова, Москва, Россия \\ 2 ООО «Генотек», Москва, Россия
}

\begin{abstract}
Развитие пренатального молекулярного скрининга анеуплоидий плода, основанного на анализе внеклеточной ДНК, циркулирующей в крови беременной, происходит бурно, особенно в последние 2-3 года. В обзоре представлены возможности и ограничения использования этой методики в клинической практике, а также причины ложноположительных и ложноотрицательных результатов скрининга. Описаны принципы, лежащие в основе технологий как получения, так и анализа данных. Рассмотрены мнения профессиональных сообществ, а также особенности законодательного регулирования применения неинвазивного пренатального скрининга (НИПС) в клинической практике в странах, где уровень использования НИПС существенно превышает отечественный.
\end{abstract}

Ключевые слова: NIPT, НИПС, пренатальный скрининг, анеуплоидии плода, внеклеточная ДНК

Благодарности: авторы очень признательны сотруднице ФГБУ «НМИЦ АГП имени В. И. Кулакова» Екатерине Шубиной за ценные замечания и рекомендации, которые она давала в ходе подготовки обзора.

Информация о вкладе авторов: Д. О. Коростин - идея и план публикации, общее руководство подготовкой публикации; Д. А. Плахина — подготовка разделов о внДНК и о законодательстве, редактирование рукописи; В. А. Белова - подготовка разделов о НИПС с помощью МРS, подготовка раздела о законодательстве, редактирование рукописи.

$\bowtie$ Для корреспонденции: Дмитрий Олегович Коростин

Наставнический переулок, д. 17, к. 1, г. Москва, 105120; d.korostin@gmail.com

Статья получена: 05.10.2018 Статья принята к печати: 10.05.2019 Опубликована онлайн: 22.05.2019

DOI: $10.24075 /$ vrgmu.2019.036

\section{NONINVASIVE PRENATAL TESTING: THE ASPECTS OF ITS INTRODUCTION INTO CLINICAL PRACTICE}

Korostin $\mathrm{DO}^{1,2}$, Plakhina $\mathrm{DA}^{2}$, Belova $\mathrm{VA}^{1,2}$

${ }_{1}^{1}$ Pirogov Russian National Research Medical University, Moscow, Russia

${ }^{2}$ Genotek Ltd., Moscow, Russia

The last couple of years have witnessed the rapid development of prenatal molecular-based screening for fetal aneuploidies that utilizes the analysis of cell-free DNA circulating in the bloodstream of a pregnant woman. The present review looks at the potential and limitations of such testing and the possible causes of false-positive and false-negative results. The review also describes the underlying principles of data acquisition and analysis the testing involves. In addition, we talk about the opinions held by the expert community and some aspects of legislation on the use of noninvasive prenatal testing (NIPT) in clinical practice in the countries where NIPT is much more widespread than in Russia.

Keywords: NIPT, NIPS, prenatal screening, fetal aneuploidy, cell-free DNA

Acknowledgment: the authors are grateful for Ekaterina Shubina of Kulakov National Medical Research Center for Obstetrics, Gynecology and Perinatology for her valuable feedback.

Author contribution: Korostin DO conceived the review and supervised manuscript preparation; Plakhina DA wrote the sections about cell-free DNA and the regulatory legislation and helped to revise the manuscript; Belova VA wrote the sections about MPS-aided NIPT and the regulatory legislation and helped to revise the manuscript.

$\triangle$ Correspondence should be addressed: Dmitry O. Korostin Nastavnichesky per. 17, str. 1, Moscow, 105120; d.korostin@gmail.com

Received: 05.10.2018 Accepted: 10.05.2019 Published online: 22.05.2019

DOI: $10.24075 /$ brsmu.2019.036

Анеуплоидии хромосом генома плода являются одной из основных (35\%) причин спонтанных выкидышей [1] и возникают с частотой до 0,3\% всех случаев рождения детей $[2,3]$. Наиболее часто у новорожденных встречаются трисомии по 13-й, 18-й, 21-й X-хромосомам и кариотип XXY.

Среди анеуплоидий плода наиболее распространен синдром Дауна (СД), он встречается с частотой 1 случай на 800 рождений [4]. Известно, что риск анеуплоидии коррелирует с возрастом матери, причем с 34 лет кривая риска приобретает экспоненциальный характер, приближаясь к частоте 1 случай на 35 родов у женщин старше 40 лет [5].

До 1980-х гг. возраст матери был единственным надежным критерием оценки риска наличия анеуплоидии, поэтому всем беременным старше 35 лет рекомендовали пройти инвазивные диагностические тесты, достоверно определяющие кариотип плода. Показанием для проведения инвазивной диагностики у более молодых беременных была лишь семейная история [6].

Сегодня наиболее совершенным скринингом считают комбинированный тест или скрининг 1-го триместра, представленный в 1997 г. [7]. Его чувствительность достигает 90\% для СД с 5\% ложноположительных результатов [8].

Для диагностирования наследственных патологий плода применяют только подходы, основанные на прямом анализе клеток плода, полученных из ворсин хориона или амниотической жидкости - инвазивная диагностика (ИД). Полученный биоматериал анализируют с помощью ряда методов: QF-PCR, MLPA, G-banding, FISH, молекулярного кариотипирования [9]. 


\section{Происхождение внеклеточной фетальной ДНК}

Внеклеточная фетальная ДНК (фДНК) преодолевает плацентарный барьер и циркулирует в кровотоке матери [10]. Современными методами фДНК может быть обнаружена в плазме крови матери, начиная с 4-й недели беременности; ее содержание возрастает на протяжении всего гестационного периода, достигая максимального пика в последние 8 недель, и резко снижается почти до нуля в первые часы после родоразрешения [11-15]. В кровь фДНК попадает в результате апоптоза клеток трофобласта плаценты [16]. Подтверждением этому служит обнаружение фДНК в случаях анэмбрионической беременности, при которой отсутствует плод и присутствует только плацентарная ткань [17], а также в случаях мейотического плацентарного мозаицизма (ПМ).

Явление ПМ (различие кариотипа плода и плаценты) наблюдается у 0,6-1\% пациентов, прошедших ИД [18]. По происхождению ПМ делят на митотический и мейотический типы. В первом случае у диплоидной зиготы в одном из делений клеток плаценты происходит нарушение расхождения хромосом, что приводит к возникновению анеуплоидной клеточной линии и явлению ограниченного ПМ. Ограниченный ПМ, как правило, локализован в определенном регионе плаценты и цитогенетически может быть определен как «мозаицизм низкого уровня». Мейотический ПМ происходит из изначально трисомной зиготы, в которой осуществляется «спасение» хромосомы за счет потери одной из ее копий на ранних этапах развития плода. Таким образом, плод может иметь нормальный кариотип при частично или абсолютно анеуплоидной плаценте и наоборот.

\section{Характеристики фДНК}

В крови матери вкДНК находится в виде фрагментов. Фрагменты материнской вкДНК преимущественно имеют длину 166 п.о., а фрагменты фДНК - 143 п.о. [19]. Такое распределение связано с неслучайной нарезкой ДНК [20]. Деградация ДНК происходит под воздействием различных ферментов и зависит от доступности участков молекуль ДНК для них. Нуклеосомы, формирующие первичный уровень компактизации ДНК в виде ее намотки на «шайбы» из гистонов, расположены на расстоянии в среднем 20 п.о. друг от друга. Наиболее доступным для нуклеаз оказывается как раз этот связывающий нуклеосомы линкерный участок. Тогда фрагменты фДНК размером 143 п.о. соответствуют длине витка ДНК вокруг нуклеосомь без линкеров с двух сторон, а фррагменты материнской вкДНК размером 166 п.о. соответствуют длине витка ДНК с «необрезанным» линкером. Неслучайная нарезка может быть связана с наличием разных форм гистона Н1 в нуклеосомах разного происхождения - плацентарного и гематопоэтического. Основная функция гистона $\mathrm{H1}$ связывание с линкером; такого связывания, видимо, не происходит в случае с фДНК, и линкер обрезается [19, 21].

«Пилообразный» профиль распределения фрагментов меньшей длины, где каждый пик отстает от другого на 10 п.о., по-видимому, свидетельствует о том, что вКДНК подвергается дальнейшему нуклеазному расщеплению в апоптотических тельцах в районе каждого 10-го нуклеотида, непосредственно прикрепленного к гистонам [19, 22]. При анализе длин коротких прочтений, картированных на митохондриальный геном, который не связан с гистонами, подобного распределения длин фраментов не наблюдается.
Установлено, что участки генома плода (и плаценты) могут быть гипо- или гиперметилированы отлично от материнских ввиду эпигенетических межтканевых различий [23, 24]. Есть гипотеза [25], что более доступными для разрезания оказываются неметилированные участки ДНК. Материнская вкДНК гиперметилирована, что приводит к более плотной намотке ДНК на гистоны, к повышению компактности и стабильности нуклеосом и увеличению средней длины фрагментов вКДНК в отличие от фДНК.

\section{НИПС с помощью MPS}

Потенициальными мишенями для проведения исследований генома плода в крови матери могут быть фетальные клетки, внеклеточная фетальная РНК (вКРНК) и фДНК. Благодаря ряду преимуществ, на анализе фДНК сегодня основаны методы неинвазивного пренатального молекулярного скрининга (НИПС).

Доля фДНК составляет в среднем 10\% всей вкДНК в актуальный для анализа период гестации, что превышает долю фетальных клеток в крови матери на 3-4 порядка.

Влияние материнского микрохимеризма, как правило, несущественно по сравнению с долей фетальной ДНК; вкДНК стабильна по сравнению с вкРНК, а методы ее анализа демонстрируют более высокую воспроизводимость в разных лабораториях.

НИПС представляет собой статистическое исследование, основная цель которого - оценить долю представленности каждой из хромосом в исследуемом образце. В норме на каждую из хромосом генома небеременной женщины будет приходиться пропорциональное ее длине количество коротких прочтений (ридов). Если женщина беременна ребенком с нормальным кариотипом, картина не изменится. Но если у ребенка имеется трисомия, например по 21-й хромосоме, то ее доля по отношению к другим хромосомам вырастет. Длина 21-й хромосомы составляет примерно 1,5\% генома. Если доля фДНК у образца 10\%, то дополнительная фетальная 21-я хромосома повысит представленность примерно на 0,08\%. Чтобы оценить достоверность полученных результатов, используют различные статистические методики оценки. Наиболее распространена методика Z-критерия Фишера. C помощью Z-теста проверяют, не является ли увеличение покрытия хромосомы случайным, для чего сравнивают ее значение с математическим ожиданием покрытия с учетом его стандартной ошибки. Расчет $Z$ проводят по следующей формуле:

$$
Z=(x-\mu) / \delta
$$

где для некоторой хромосомы А $x$ - это число ридов, картированных на нее в анализируемом образце; $\mu-$ среднее значение числа ридов, относящихся к А, полученных от анализа референсных образцов (нормальный контроль); $\delta$ - стандартное отклонение. Результирующее значение $Z$-теста > 3 считают признаком трисомии, значение $<-3$ указывает на моносомию, а значения в диапазоне от -3 до 3 - на нормальный кариотип [26].

Математическое ожидание вычисляют с помощью проведенного заранее анализа выборки образцов вкДНК с известным диагнозом у ребенка.

Отметим еще раз, что физически вкДНК матери не отделяют от вКДНК плода, а исследуют совокупную ВКДНК. Поэтому в случае многоплодной беременности НИПС может определить наличие анеуплоидии у одного из близнецов, но не укажет на ребенка с патологией. 
Важным критерием, влияющим на НИПС, является доля фетальной вкДНК. Чем она больше, тем большее значение $Z$ покажет анализ в случае анеуплоидии. Необходимый минимум содержания фетальной фракции для проведения НИПС оценивают в 4\% [27-29].

Существует множество способов оценки доли фДНК. Их объединяет принцип поиска значимых отличий фетальной фракции вкДНК от материнской. Наиболее очевидна оценка по доле Ү-хромосомы, значение которой составляет половину значения фДНК. Этот подход применим только в случае вынашивания мальчика.

Универсальна и широко употребима оценка по SNP. Идея проста: необходимо найти такие полиморфизмы, по которым мать и ребенок будут иметь разные генотипь (мать - гомозигота, а ребенок - гетерозигота за счет отцовского аллеля). Регионы с полиморфизмами необходимо многократно прочитать, а затем оценить количество ридов, пришедшееся на отцовский аллель [30-32]. Умножив на 2 долю ридов, получим долю фДНК. Подбор SNP осуществляют по следующим критериям:

- имеют частоту минорного аллеля (minor allele frequency, MAF), близкую к 50\%;

- входят в разные группы сцепления;

• не подвергаются отбору.

В пределе, увеличивая количество анализируемых SNP, можно определять анеуплоидии, сравнивая долю покрытия фетальных и материнских хромосом по соотношению ридов соответствующих SNP. Подобным образом поступила компания Natera, НИПС у которой основан на анализе почти 20000 SNP [33].

Было показано, что оценка доли участков с дифференциальным уровнем метилирования среди результатов анализа внеклеточного метилома позволяет надежно оценивать долю фДНК [34].

Так как распределение длин плодных и материнских молекул вкДНК неодинаково, определять долю фетальной вкДНК можно, базируясь на соотношении между количеством фрагментов в диапазоне от 100 до 150 п.о. и от 163 до 169 п.о., соответствующих фетальной фрракции и материнской [35]. Технически такой подход эффективен при использовании pair-end чтений в процессе секвенирования [36].

Еще один метод оценки доли фретальной ДНК по «нуклеосомному треку" находится в разработке. Идея метода поддерживается тем фактом, что фетальная ДНК фрагментирована не случайным образом, а в соответствии с упаковкой нуклеосом, как было указано выше [37].

Для оценки доли фетальной ДНК стали активно привлекать нейронные сети. Имея достаточно большие обучающие выборки (тысячи образцов с известной долей фДНК), можно добиться достоверной оценки доли по определенной совокупности параметров секвенирования [38].

\section{Возможности НИПС}

В большинстве случаев НИПС используют для поиска анеуплоидий хромосом, однако технически возможности MPS позволяют определять и другие нарушения в геноме.

Секвенирование генома с низким и сверхнизким (менее ×1,0) покрытием не позволяет обнаруживать точковые мутации, однако вполне применимо для поиска делеций и дупликаций [39]. Именно таким образом проводят пренатальный генетический скрининг методами высокопроизводительного секвенирования [40]. Разрешающая способность данных НИПС в большинстве случаев недостаточна для поиска среднего размера делеций и дупликаций (до 5 млн п.о.), что можно решить увеличением количества получаемых данных на каждый образец [41-44]. Этот подход ведет к существенному увеличению себестоимости НИПС. Несколько менее эффективными оказываются более сложные способы биоинформатической обработки данных секвенирования $[45,46]$. Однако благодаря ряду технологий обогащения библиотек геномной ДНК можно значительно повысить долю данных секвенирования, приходящихся на интересующие регионы. Например, в тесте Panorama [47] используют процедуру обогащения генома примерно до 20000 локусов, имеющих более плотное расположение в областях микроделеционных синдромов. Благодаря такому подходу, по мнению разработчиков, удается выявлять микроделеции с точностью 97,8\% и выше [48].

С момента открытия фДНК были разработаны подходы для диагностики генетических нарушений у плода. Первые среди них, способы определения пола ребенка [49] и его резус-статуса [50], основаны на поиске нехарактерных для материнского генома последовательностей с помощью различных ПЦР (qPCR, ddPRC, QF-PCR). В дальнейшем разработка методик диагностики признаков, наследуемых от отца, стала носить рутинный характер: появились STRмаркеры X-хромосомы, унаследованной от отца [51], а также маркеры аутосомно-доминантных заболеваний, например хореи Гентингтона [52] и миотонической дистрофии [53]. Однако большинство моногенных заболеваний аутосомнорецессивны и развиваются при наличии мутаций в обеих копиях гена. Поэтому на сегодняшний день используют три секвенирования: секвенируют геномную ДНК матери и отца, чтобы определить гаплотипы, а также в каком из них локализованы интересующие мутации, а затем секвенируют вкДНК, в которой уже по маркерам гаплотипов определяют, какие конкретно хромосомы унаследовал развивающийся ребенок [54].

Метиломный анализ внеклеточного генома плода показал наличие паттерна метилирования, по которому можно определить анеуплоидию $[55,56]$. Также выявлено, что метилом плаценты (а ведь в НИПС анализируют именно его) динамичен, паттерн метилирования может меняться в зависимости от клинического состояния плода и матери. Так, метиломный анализ вкДНК может послужить методом диагностики преэклампсии [57-59].

Несмотря на сложности в работе с внРНК (контаминация неинформативной рРНК, плохая сохранность в биоматериале, более низкая воспроизводимость результатов по сравнению с вкДНК), показано, что изменение экспрессии некоторых транскриптов в фетальном транскриптоме может служить хорошим маркером развития той же преэклампсии еще до проявления клинических симптомов болезни [60].

\section{Валидация НИпС}

Несмотря на возможности НИПС, методика должна была пройти ряд клинических испытаний, которые докажут ее эффективность.

В 2014 г. на выборке 1914 беременных из 21 медицинского центра США было показано, что частота ложноположительных результатов НИПС по сравнению со стандартным биохимическим скринингом была существенно ниже (0,3\% против 3,6\% при p < 0,001 - для Т21 и 0,2\% против 0,6\% при $p<0,03-$ для Т18). Стоит отметить, что для 0,9\% пациентов получить результаты НИПС не удалось [61]. 
В 2015 г. было опубликовано обширное исследование по сравнению эффективности НИПС с традиционными методиками [62], проводимое в 35 медицинских центрах на выборке из 15841 беременной. Для Т21 с помощью НИПС удалось обнаружить все случаи (38) истинной анеуплоидии, а у 9 пациенток результат оказался ложноположительным. Таким образом, DR для T21 составила 100\%, FPR - 0,06\%, PPV - 80,9\% (значения стандартного скринига на этой выборке составили 78,9\%, 5,4\%, 3,4\% соответственно). По Т13 и Т18 результаты НИПС также оказались существенно лучше стандартного скрининга. Таким образом, НИПО можно использовать для выявления трисомий у плода, так как он имеет более высокое разрешение и точность по сравнению с традиционными подходами.

\section{Причины ложных результатов НИПС}

НИПС имеет ряд ограничений, которые могут приводить к ошибочным результатам.

\section{Вес матери и срок гестации}

Количество фДНК находится в прямой зависимости от срока гестации и в обратной — от ИМТ беременной. Именно низкая доля ффДНК на сроках менее 9-10 недель беременности не позволяет получать надежные результаты НИПС. Для женщин с высоким ИМТ проведение НИПС также может оказаться неэфективным, так как вероятность ложного результата будет высокой $[15,63]$ без оценки доли фДНК.

\section{Плацентарный мозаицизм}

Для исключения вероятности наличия ПМ наиболее предпочтительным способом забора материала для инвазивной диагностики после положительного НИПО является амниоцентез. Биопсия ворсин хориона может быть непоказательна, так как ДНК в них имеет такое же плацентарное происхождение, как и фДНК [64-67]. Явление ПМ - еще один признак необходимости подтверждения диагноза, обнаруженного НИПС, инвазивным способом. Решение о прерывании беременности на основании только результатов НИПС принимать категорически нельзя (см. раздел ниже).

\section{Близнецы}

НИПС может быть использован для анализа анеуплоидий и при двуплодных беременностях, однако тест не позволяет определить, какой из близнецов имеет анеуплоидию, это необходимо делать с помощью инвазивной диагностики обоих детей. Несмотря на повышенную долю общей фДНК по сравнению с одноплодными беременностями [68] и возможность ее оценки для каждого из близнецов, точность теста снижена по сравнению с одноплодными беременностями [69].

Среди многоплодных беременностей встречается явление исчезающего близнеца, при котором развитие одного из плодов замирает в первом триместре беременности. Показано, что частота анеуплоидий среди исчезающих близнецов существенно выше, чем при нормальном развитии обоих плодов. Так как НИПС анализирует всю вКДНК и в большинстве случаев не позволяет определять наличие дополнительных гаплотипов в образцах, исчезающий близнец может и влиять на ложноположительный результат, когда сам является анеуплоидом, и маскировать анеуплоидию второго близнеца, приводя к ложноотрицательным результатам НИПС, и мешать корректному определению пола ребенка. Согласно оценке более 30000 НИПС, частота встречаемости исчезающих близнецов с анеуплоидиями составляет 0,11\% среди всей выборки [70]. Эти данные близки к значению ложноположительных результатов, полученных в ходе крупного метаанализа публикаций, посвященных НИПС [71]. Чтобы избежать ошибок при НИПС, связанных с исчезающим близнецом, стоит уделять более пристальное внимание УЗИ-исследованию, проводимому в І триместре, в котором можно обнаружить второй плод.

\section{CNV у родителей}

По некоторым данным, причиной 17\% ложноположительных результатов НИПС были CNV в материнских клетках на соответствующих хромосомах размером от 0,5 млн до 14 млн п.о. [72].

Мозаицизм у родителей также может стать причиной ложных результатов НИПС, как и отмеченный ранее ПМ. Например, частота моносомии по X прямо коррелирует с возрастом женщины [74], и было показано, что 16\% обнаруженных с помощью НИПС анеуплоидий плода по половым хромосомам на самом деле связаны с нарушениями в кариотипе матери по Х-хромосоме [65]. В зависимости от определения нижней границы мозаицизма, частота встречаемости моносомий по X составляет от 1 : 3300 (доля мозаичных клеток выше 34\%) [74] до 1 : 300 (доля мозаичных клеток 4\% и выше) [75].

\section{Опухоли}

Показано, что различные формы онкологических заболеваний беременной приводят к искажению результатов НИПС, так как клеткам опухолей присуща геномная нестабильность, опухоли, как правило, высокоангиогенизированы и выделяют большое количество вкДНК в просвет сосудистого русла [76].

\section{Заблуждение по поводу опасности проведения ИД}

В качестве одного из аргументов, приводимых за более активное внедрение НИПС в клиническую практику, компании — производители услуг НИПС называют опасность осложнений (включая потерю плода), возникающих при проведении процедуры забора биоматериала: оба подхода забора биоматериала ребенка примерно в 1\% случаев приводят к потере беременности [4, 77, 78]. Однако другие авторы приводят более низкие значения потери: для ИД $1: 200$ для забора ворсин хориона и $1: 300$ для амниоцентеза [79, 80]. Эти значения ниже, чем в случаях самопроизвольного прерывания беременности [81].

\section{Законодательство и рекомендации для НИПС}

На сегодняшний день во многих странах главенствуют две модели внедрения НИПС в клиническую практику.

1. Контингентная: тест назначают женщинам из группы риска по результатам скрининга, проводимого в І триместре. Такой подход позволяет субсидировать оплату части или всей стоимости НИПС из государственного бюджета.

2. Коммерческая: тест предлагают проходить беременным, имеющим финансовые (собственные или за счет медицинской страховки) ресурсы для его оплаты. 
В Нидерландах и Дании кроме того проводят широкомасштабные исследования применимости НИПС TRIDENT-2 (http://www.meerovernipt.nl), в рамках которых беременным предлагают пройти НИПС вместо скрининга в I триместре.

Ниже рассмотрены примеры использования и регулирования НИПС в некоторых странах.

\section{Великобритания}

Ежегодно в этой стране регистрируют 800000 случаев беременностей. В стране действует Национальный скрининговый комитет [82], который в январе 2016 г. опубликовал рекомендации по инкорпорированию НИПС в существующую программу выявления аномалий развития плода [83]. Согласно этому документу, НИПС рекомендуется проводить всем женщинам, имеющим повышенный (> 1: 150) риск анеуплоидий по результатам комбинированного теста, проводимого на 10-14-й неделе беременности. Проведение оценки эффективности методики запланировано на 2018-2019 гг. В случае успеха, расходы на проведение НИПС будут как минимум частично покрываться за счет сокращения числа ИД и высвобождения выделенных на них средств.

\section{Швеция}

Ежегодно в этой стране насчитывают 120000 случаев беременностей. Шведское общество акушеров и гинекологов в июне 2016 г. выпустило руководство [84], в котором рекомендует проводить НИПС всем женщинам имеющим по результатам комбинированного скрининга промежуточное значение риска анеуплоидий (от $1: 51$ до 1 : 1000), а также при ограничениях проведения ИД (инфицирование беременной ВИЧ или гепатитом). С осторожностью необходимо назначать НИПС при многоплодных беременностях. В случае более высокого риска необходимо проведение ИД, более низкого стандартного мониторинга беременности. Общество не рекомендует проводить НИПС всем беременным по причине недостаточных доказательств эффективности теста для всех групп этой категории пациенток и высокой стоимости исследования.

\section{Франция}

Во Франции количество забеременевших женщин достигает около 800000 человек в год. В 2017 г. Министерство здравоохранения Франции выпустило свои рекомендации [85]. До НИПС система выявления анеуплоидий была основана на стандартах FMF [29]. В случае определения высокого риска (> 1 : 250), проводили ИД с последующим кариотипированием. Все процедуры оплачивает система страхования здоровья Франции. Согласно рекомендациям 2017 г., анализ циркулирующей вкДНК рекомендован при проведении скрининга на трисомию по 21-й хромосоме для женщин, имеющих повышенный риск по результатам комбинированного скрининга, проводимого в I триместре (от 1 : 1000 до 1 : 51). Для беременных с риском 1 : 50 и выше рекомендовано проводить ИД, однако женщины могут выбрать сначала и проведение молекулярного скрининга. Особо отмечено, что НИПС не следует рассматривать как замену ИД. Рекомендуется также разработать систему контроля качества и аккредитации лабораторий, проводящих НИПС. Через 3 года запланирован пересмотр стратегии скрининга, включая вопросы о скрининге на другие анеуплоидии и микроделеции.

\section{США}

В США ежегодно регистрируют около 6,35 млн беременностей. Рынок НИПС полностью коммерциализирован и принадлежит нескольким крупным компаниям (табл.) [86].

Оплата НИПС происходит либо за счет страховых компаний, либо из собственных средств пациентов. Государство НИПС не субсидирует.

В настоящее время выпущено четыре актуальных рекомендации:

- Американского общества акушеров и гинекологов (ACOG) в мае 2016 г. [87];

- Международного общества по пренатальной диагностике (ISPD) в апреле 2015 г. [80];

• Национального общества генетических консультантов (NSGC) в октябре 2016 г. [88];

- Американской коллегии по медицинской генетике и геномике (ACMG) [89].

В документе ACMG отмечено, что развитие техник и методик анализа в области НИПС происходит так быстро, что любые клинические указания и директивы устаревают буквально за считанные годы. Как и в вышедших чуть раньше рекомендациях ACOG, в руководстве ACMG указано, что все беременные женщины должны быть соответствующим образом проинформированы о возможности проведения НИПС и его относительных преимуществах перед традиционным скринингом в выявлении анеуплоидий по 13-й, 18-й и 21-й хромосомам. Некоторые специалисты и компании интерпретировали содержимое как рекомендацию по назначению НИПС для всех беременных женщин, независимо от риска, показанного на скрининге в I триместре. Это не соответствует действительности. Фактически АCMG рекомендует информировать всех женщин о наличии НИПС, но только с указанием на соответствующую информационную и директивную поддержку [86]. К сожалению, до сих пор многие независимые врачи недостаточно осведомлены об ограничениях НИПС, некорректно интерпретируют результаты исследований и принимают ошибочные решения. Зная об этом, компании проводят собственные медико-генетические консультирования, к которым у регуляторов есть вопросы, так как врачи, их осуществляющие, могут быть ангажированы.

Отмечено возрастающее число ложноположительных случаев, связанных с определением анеуплоидий по

Таблица. Ведущие компании США, предлагающие коммерческие НИПС

\begin{tabular}{|c|c|c|}
\hline Коммерческое название теста & Компания & Расположение \\
\hline MaterniT21Plus $^{\text {TM }}$ & Sequenom, «дочка» LabCorp, Inc. $^{\text {San Diego, CA }}$ \\
\hline Verifi $^{\text {TM }}$ & Verinata Health, теперь Illumina & Redwood City, CA \\
\hline Harmony $^{\text {TM }}$ & Ariosa Diagnostics & San Jose, CA \\
\hline Panorama $^{\text {TM }}$ & Natera & San Carlos, CA \\
\hline
\end{tabular}


половым хромосомам. В связи с этим указывается на обязательное информирование пациентов об этом, а также о разнообразии исходов для детей с такими анеуплоидиями. Например, кариотип ХО является распространенной причиной потери беременности, с одной стороны, а с другой, женщины с синдромом Шершевского-Тернера имеют достаточно высокое качество жизни.

Особую важность авторы рекомендаций уделяют содержимому заключения по исследованию. Для всех анализируемых типов мутаций (анеуплоидии аутосом, половых хромосом, CNV) должны быть четко прописаны параметры чувствительности, специфичности, PPV и NPV, а также доля фетальной ДНК.

Наиболее частой причиной неудачи проведения НИПС оказывается низкая доля фДНК. Показано, что низкая доля фДНК коррелирует с разными анеуплоидиями плода [62, 72], поэтому в случае неудачи рекомендуется не повторять НИПС, а сразу отправлять беременную на ИД. Комиссия ACMG не рекомендует проводить анализ микроделеций, так как хороших независимых оценок чувствительности и специфичности этого типа НИПС не проведено.

\section{Россия}

В России происходит около 1,8 млн беременностей ежегодно. Система скрининга и диагностики генетических аномалий развития плода включает в себя биохимический и УЗИ-скрининг, проводимые в I триместре, по результатам которых в случае превышения риска 1 : 100 беременная направляется на медико-генетическую консультацию и ИД. Эти процедуры финансируются за счет средств ОМС и региональных бюджетов [90]. В 2016 г. были опубликованы клинические рекомендации по проведению НИПС [91], которые во многом согласуются с описанными выше рекомендациями ACMG.

Внедрение НИПС на российском рынке в значительной мере затруднено. НИПС не имеет официальной сертификации, а практически все оборудование и реагенты, необходимые для проведения MPS, не имеют регистрационных удостоверений.

\section{ЗАКЛЮЧЕНИЕ}

В масштабах страны использование НИПС представляет собой «палку о двух концах»: если слишком поднять значение риска, выше которого следует проводить НИПС, специалисты, проводящие ИД, лишатся львиной доли своей практики, что приведет к снижению их квалисикации, и, как следствие, ошибкам ИД (т. е. статистика выявляемости может даже ухудшиться). Если наоборот «распустить» риск вплоть до назначения НИПС всем беременным, статья расходов на этот вид исследований станет очень значительной даже для самых развитых стран. Таким образом, необходимо найти то оптимальное значение риска, при котором соотношение выявляемости анеуплоидий и стоимости их обнаружения окажется оптимальным для государства.

\section{Литература}

1. Hassold $\mathrm{T}, \mathrm{Hall} \mathrm{H}$, Hunt P. The origin of human aneuploidy: where we have been, where we are going. Human molecular genetics. 2007; 16 (2): 203-8.

2. Driscoll DA, Gross S. Prenatal screening for aneuploidy. New England Journal of Medicine. 2009; 360 (24): 2556-62.

3. Nagaoka SI, Hassold TJ, Hunt PA. Human aneuploidy: mechanisms and new insights into an age-old problem. Nature Reviews Genetics. 2012; 13 (7): 493-504.

4. Ehrich M, Deciu C, Zwiefelhofer T, Tynan JA, Cagasan L, Tim R, et al. Noninvasive detection of fetal trisomy 21 by sequencing of DNA in maternal blood: a study in a clinical setting. American journal of obstetrics and gynecology. 2011; 204 (3): 205-e1.

5. Morris JK, Mutton DE, Alberman E. Revised estimates of the maternal age specific live birth prevalence of Down's syndrome. Journal of medical screening. 2002; 9 (1): 2-6.

6. Buckley F, Buckley S. Wrongful deaths and rightful lives-screening for Down syndrome. Down Syndrome Research and Practice. 2008; 12 (2): 79-86.

7. Wald NJ, Hackshaw AK. Combining ultrasound and biochemistry in first-trimester screening for Down's syndrome. Prenatal diagnosis. 1997; 17 (9): 821-9.

8. Sillence KA, Madgett TE, Roberts LA, Overton TG, Avent ND. Non-invasive screening tools for Down's syndrome: a review. Diagnostics. 2013; 3 (2): 291-314.

9. Choy KW, Kwok YK, Cheng YKY, Wong KM, Wong HK, Leung KO, et al. Diagnostic accuracy of the BACs-on-Beads ${ }^{\mathrm{TM}}$ assay versus karyotyping for prenatal detection of chromosomal abnormalities: a retrospective consecutive case series. BJOG: An International Journal of Obstetrics \& Gynaecology. 2014; 121 (10): 1245-52.

10. Lo YD, Corbetta N, Chamberlain PF, Rai V, Sargent IL, Redman CW, et al. Presence of fetal DNA in maternal plasma and serum. The lancet. 1997; 350 (9076): 485-7.

11. Lo YD, Hjelm NM, Fidler C, Sargent IL, Murphy MF, Chamberlain PF, et al. Prenatal diagnosis of fetal RhD status by molecular analysis of maternal plasma. New England Journal of Medicine. 1998; 339 (24): 1734-8.

12. Farina A, Caramelli E, Concu M, Sekizawa A, Ruggeri R, Bovicelli L, et al. Testing normality of fetal DNA concentration in maternal plasma at 10-12 completed weeks' gestation: a preliminary approach to a new marker for genetic screening. Prenatal Diagnosis: Published in Affiliation With the International Society for Prenatal Diagnosis. 2002; 22 (2): 148-52.

13. Bischoff FZ, Lewis DE, Simpson JL. Cell-free fetal DNA in maternal blood: kinetics, source and structure. Human reproduction update. 2005; 11 (1): 59-67.

14. Wang E, Batey A, Struble C, Musci T, Song K, Oliphant A. Gestational age and maternal weight effects on fetal cell-free DNA in maternal plasma. Prenatal diagnosis. 2013; 33 (7): 662-6.

15. Curnow KJ, Gross SJ, Hall MP, Stosic M, Demko Z, Zimmermann B, et al. Clinical experience and follow-up with large scale singlenucleotide polymorphism-based noninvasive prenatal aneuploidy testing. American journal of obstetrics and gynecology. 2014; 211 (5): 527-e1.

16. Tjoa ML, Cindrova-Davies T, Spasic-Boskovic O, Bianchi DW Burton GJ. Trophoblastic oxidative stress and the release of cellfree feto-placental DNA. The American journal of pathology. 2006; 169 (2): 400-4.

17. Alberry M, Maddocks $\mathrm{D}$, Jones $\mathrm{M}$, Abdel Hadi M, Abdel-Fattah $\mathrm{S}$, Avent N, Soothill PW. Free fetal DNA in maternal plasma in anembryonic pregnancies: confirmation that the origin is the trophoblast. Prenatal Diagnosis: Published in Affiliation With the International Society for Prenatal Diagnosis. 2007; 27 (5): 415-8.

18. Gardner RM, Sutherland GR, Shaffer LG. Chromosome abnormalities and genetic counseling. Oxford University Press USA. 2011; (61).

19. Lo YD, Chan KA, Sun H, Chen EZ, Jiang P, Lun FM, et al. Maternal plasma DNA sequencing reveals the genome-wide genetic and mutational profile of the fetus. Science translational medicine. 2010; 2 (61): 61ra91-61ra91.

20. Ivanov M, Baranova A, Butler T, Spellman P, Mileyko V. Nonrandom fragmentation patterns in circulating cell-free DNA reflect epigenetic regulation. BMC genomics. 2015; 16 (13): S1. 
21. Sancho M, Diani E, Beato M, Jordan A. Depletion of human histone $\mathrm{H} 1$ variants uncovers specific roles in gene expression and cell growth. PLoS genetics. 2008; 4 (10): e1000227.

22. Jiang $P$, Lo YMD. The long and short of circulating cell-free DNA and the ins and outs of molecular diagnostics. Trends in Genetics. 2016; 32 (6): 360-71.

23. Geiman TM, Muegge K. DNA methylation in early development. Molecular Reproduction and Development: Incorporating Gamete Research. 2010; 77 (2): 105-13.

24. Sun $\mathrm{K}$, et al. Plasma DNA tissue mapping by genome-wide methylation sequencing for noninvasive prenatal, cancer, and transplantation assessments. Proceedings of the National Academy of Sciences. 2015; 112 (40): E5503-E5512.

25. Sun $\mathrm{K}$, Jiang $\mathrm{P}$, Wong $\mathrm{Al}$, Cheng $\mathrm{YK}$, Cheng $\mathrm{SH}$, Zhang $\mathrm{H}$, et al. Size-tagged preferred ends in maternal plasma DNA shed light on the production mechanism and show utility in noninvasive prenatal testing. Proceedings of the National Academy of Sciences. 2018; 115 (22): E5106-E5114

26. Tamminga S, van Maarle M, Henneman L, Oudejans CB, Cornel MC Sistermans EA. Maternal plasma DNA and RNA sequencing for prenatal testing. Advances in clinical chemistry. 2016; (74): 63-102.

27. Ashoor G, Poon L, Syngelaki A, Mosimann B, Nicolaides KH Fetal fraction in maternal plasma cell-free DNA at 11-13 weeks' gestation: effect of maternal and fetal factors. Fetal diagnosis and therapy. 2012; 31 (4): 237-43.

28. Canick JA, et al. The impact of maternal plasma DNA fetal fraction on next generation sequencing tests for common fetal aneuploidies. Prenatal diagnosis. 2013; 33 (7): 667-74.

29. Wright D, Syngelaki A, Bradbury I, Akolekar R, Nicolaides KH. First-trimester screening for trisomies 21, 18 and 13 by ultrasound and biochemical testing. Fetal diagnosis and therapy. 2014; 35 (2): 118-26.

30. Kim SK, Hannum G, Geis J, Tynan J, Hogg G, Zhao C, et al. Determination of fetal DNA fraction from the plasma of pregnant women using sequence read counts. Prenatal diagnosis. 2015 35 (8): 810-15

31. Jiang $P$, Peng $X$, Su $X$, Sun $K$, Stephanie CY, Chu Wl, et al. FetalQuant SD: accurate quantification of fetal DNA fraction by shallow-depth sequencing of maternal plasma DNA. NPJ genomic medicine. 2016; (1): 16013.

32. Peng XL, Jiang P. Bioinformatics approaches for fetal DNA fraction estimation in noninvasive prenatal testing. International journal of molecular sciences. 2017; 18 (2): 453.

33. Zimmermann B, Hill M, Gemelos G, Demko Z, Banjevic M, Baner J, et al. Noninvasive prenatal aneuploidy testing of chromosomes $13,18,21, X$, and $Y$, using targeted sequencing of polymorphic loci. Prenatal diagnosis. 2012; 32 (13): 1233-41.

34. Lun FM, Chiu RW, Sun K, Leung TY, Jiang P, Chan KA, et al Noninvasive prenatal methylomic analysis by genomewide bisulfite sequencing of maternal plasma DNA. Clinical chemistry. DOI: 10.1373/clinchem.2013.212274.

35. Yu SC, et al. Size-based molecular diagnostics using plasma DNA for noninvasive prenatal testing. Proceedings of the National Academy of Sciences. 2014; 111 (23): 8583-8.

36. Cirigliano V, Ordoñez E, Rueda L, Syngelaki A, Nicolaides KH. Performance of the neoBona test: a new paired-end massively parallel shotgun sequencing approach for cell-free DNA-based aneuploidy screening. Ultrasound in Obstetrics \& Gynecology. 2017; 49 (4): 460-4.

37. Straver R, Oudejans C, Sistermans EA, Reinders MJ. Calculating the fetal fraction for noninvasive prenatal testing based on genome-wide nucleosome profiles. Prenatal diagnosis. 2016; 36 (7): 614-21.

38. Kim SK, Hannum G, Geis J, Tynan J, Hogg G, Zhao C, Boom D. Determination of fetal DNA fraction from the plasma of pregnant women using sequence read counts. Prenatal diagnosis. 2015 35 (8): 810-5.

39. Chen S, Lau TK, Zhang C, Xu C, Xu Z, Hu P, et al. A method for noninvasive detection of fetal large deletions/duplications by low coverage massively parallel sequencing. Prenatal diagnosis. 2013; 33 (6): 584-90.

40. Van den Veyver IB. Recent advances in prenatal genetic screening and testing. F1000 Research. 2016; 5 (F1000 Faculty Rev): 2591
41. Peters D, Chu T, Yatsenko SA, Hendrix N, Hogge WA, Surti U, et al. Noninvasive prenatal diagnosis of a fetal microdeletion syndrome. New England Journal of Medicine. 2011; 365 (19): 1847-8.

42. Jensen TJ, Dzakula Z, Deciu C, van den Boom D, Ehrich M Detection of microdeletion 22q11. 2 in a fetus by next-generation sequencing of maternal plasma. Clinical chemistry. 2012; 58 (7): 1148-51.

43. Srinivasan A, Bianchi DW, Huang $H$, Sehnert AJ, Rava RP. Noninvasive detection of fetal subchromosome abnormalities via deep sequencing of maternal plasma. The American Journal of Human Genetics. 2013; 92 (2): 167-76.

44. Lefkowitz RB, Tynan JA, Liu T, Wu Y, Mazloom AR, Almasri E, et al. Clinical validation of a noninvasive prenatal test for genomewide detection of fetal copy number variants. American journal of obstetrics and gynecology. 2016; 215 (2): 227-e1.

45. Straver R, Sistermans EA, Holstege H, Visser A, Oudejans CB, Reinders MJ. WISECONDOR: detection of fetal aberrations from shallow sequencing maternal plasma based on a within-sample comparison scheme. Nucleic acids research. 2013; 42 (5): e31-e31.

46. Zhao C, Tynan J, Ehrich M, Hannum G, McCullough R, Saldivar JS, Deciu C. Detection of fetal subchromosomal abnormalities by sequencing circulating cell-free DNA from maternal plasma. Clinical chemistry. 2015.

47. Nicolaides KH, Syngelaki A, Gil M, Atanasova V, Markova D. Validation of targeted sequencing of single-nucleotide polymorphisms for non-invasive prenatal detection of aneuploidy of chromosomes 13, 18, 21, X, and Y. Prenatal diagnosis. 2013; 33 (6): 575-9.

48. Wapner RJ, Babiarz JE, Levy B, Stosic M, Zimmermann B, Sigurjonsson S, Hu J. Expanding the scope of noninvasive prenatal testing: detection of fetal microdeletion syndromes. American journal of obstetrics and gynecology. 2015; 212 (3): 332-e1.

49. Costa JM, Benachi A, Gautier E. New strategy for prenatal diagnosis of X-linked disorders. New England Journal of Medicine. 2002; 346 (19): 1502.

50. Lo YD, Hjelm NM, Fidler C, Sargent IL, Murphy MF, Chamberlain PF, et al. Prenatal diagnosis of fetal RhD status by molecular analysis of maternal plasma. New England Journal of Medicine. 1998; 339 (24): 1734-8.

51. Tang NL, Leung TN, Zhang J, Lau TK, Lo YD. Detection of fetalderived paternally inherited X-chromosome polymorphisms in maternal plasma. Clinical chemistry. 1999; 45 (11): 2033-5.

52. Bustamante-Aragonés A, de Alba MR, Perlado S, Trujillo-Tiebas MJ, Arranz JP, Díaz-Recasens J, et al. Non-invasive prenatal diagnosis of single-gene disorders from maternal blood. Gene. 2012; 504 (1): 144-9.

53. Amicucci P, Gennarelli M, Novelli G, Dallapiccola B. Prenatal diagnosis of myotonic dystrophy using fetal DNA obtained from maternal plasma. Clinical chemistry. 2000; 46 (2): 301-2.

54. Vermeulen C, Geeven G, de Wit E, Verstegen MJ, Jansen RP, van Kranenburg $M$, et al. Sensitive monogenic noninvasive prenatal diagnosis by targeted haplotyping. The American Journal of Human Genetics. 2017; 101 (3): 326-39.

55. Tong YK, Jin S, Chiu RW, Ding C, Chan KA, Leung TY, et al. Noninvasive prenatal detection of trisomy 21 by an epigeneticgenetic chromosome-dosage approach. Clinical chemistry. 2010; 56 (1): 90-8.

56. Tsui DW, Lam YD, Lee WS, Leung TY, Lau TK, Lau ET, et al. Systematic identification of placental epigenetic signatures for the noninvasive prenatal detection of Edwards syndrome. PloS one. 2010; 5 (11): e15069.

57. Yuen RK, Penaherrera MS, Von Dadelszen P, McFadden DE, Robinson WP. DNA methylation profiling of human placentas reveals promoter hypomethylation of multiple genes in early-onset preeclampsia. European Journal of Human Genetics. 2010; 18 (9): 1006.

58. Blair JD, Yuen RK, Lim BK, McFadden DE, von Dadelszen P, Robinson WP. Widespread DNA hypomethylation at gene enhancer regions in placentas associated with early-onset preeclampsia. Molecular human reproduction. 2013; 19 (10): 697-708.

59. Chu T, Bunce K, Shaw P, Shridhar V, Althouse A, Hubel C, et al. Comprehensive analysis of preeclampsia-associated DNA methylation in the placenta. PLoS One. 2014; 9 (9): e107318. 
60. Hahn S, Rusterholz C, Hösli I, Lapaire O. Cell-free nucleic acids as potential markers for preeclampsia. Placenta. 2011; (32): S17-S20.

61. Bianchi DW, Parker RL, Wentworth J, Madankumar R, Saffer C, Das $A F$, et al. DNA sequencing versus standard prenatal aneuploidy screening. New England journal of medicine. 2014; 370 (9): 799-808.

62. Norton ME, Jacobsson B, Swamy GK, Laurent LC, Ranzini AC, Brar $\mathrm{H}$, et al. Cell-free DNA analysis for noninvasive examination of trisomy. New England Journal of Medicine. 2015; 372 (17): 1589-97.

63. Wataganara T, Peter I, Messerlian GM, Borgatta L, Bianchi DW. Inverse correlation between maternal weight and second trimester circulating cell-free fetal DNA levels. Obstetrics \& Gynecology. 2004; 104 (3): 545-50

64. Румянцев А. Г., Курцер М. А., Мареева Ю. М., Мисюрин А. В., Румянцев С. А., Устюгов А. Ю. Клиническое значение фетального микрохимеризма у матери. Гены и клетки. 2012; 7 (2): 103-111.

65. Wang $Y$, et al. Maternal mosaicism is a significant contributor to discordant sex chromosomal aneuploidies associated with noninvasive prenatal testing. Clinical chemistry. 2014; 60 (1): 251-9.

66. Bianchi DW. Cherchez la femme: maternal incidental findings can explain discordant prenatal cell-free DNA sequencing results. Genetics in Medicine. 2017; DOI: 10.1038/gim.2017.219.

67. Hartwig TS, Ambye L, Sørensen S, Jørgensen FS. Discordant non-invasive prenatal testing (NIPT)-a systematic review. Prenatal diagnosis. 2017; 37 (6): 527-39.

68. Attilakos G, Maddocks DG, Davies T, Hunt LP, Avent ND, Soothill PW, et al. Quantification of free fetal DNA in multiple pregnancies and relationship with chorionicity. Prenatal diagnosis. 2011; 31 (10): 967-72.

69. Bevilacqua E, Gil MM, Nicolaides KH, Ordoñez E, Cirigliano V, Dierickx $\mathrm{H}$, et al. Performance of screening for aneuploidies by cell-free DNA analysis of maternal blood in twin pregnancies. Ultrasound in Obstetrics \& Gynecology. 2015; 45 (1): 61-6.

70. Curnow KJ, Wilkins-Haug L, Ryan A, Kırkızlar E, Stosic M, Hall MP, et al. Detection of triploid, molar, and vanishing twin pregnancies by a single-nucleotide polymorphism-based noninvasive prenatal test. American journal of obstetrics and gynecology. 2015; 212 (1): 79-e1.

71. Gil MM, Quezada MS, Revello R, Akolekar R, Nicolaides KH. Analysis of cell-free DNA in maternal blood in screening for fetal aneuploidies: updated meta-analysis. Ultrasound in obstetrics \& gynecology. 2015; 45 (3): 249-66.

72. Zhang $H$, Gao $Y$, Jiang F, Fu M, Yuan $Y$, Guo $Y$, et al. Noninvasive prenatal testing for trisomies 21, 18 and 13: clinical experience from 146958 pregnancies. Ultrasound in Obstetrics \& Gynecology. 2015; 45 (5): 530-8.

73. Russell LM, Strike P, Browne CE, Jacobs PA. X chromosome loss and ageing. Cytogenetic and genome research. 2007; 116 (3): 181-5.

74. Samango-Sprouse C, Kırkızlar E, Hall MP, Lawson P, Demko Z, Zneimer SM, et al. Incidence of $X$ and $Y$ chromosomal aneuploidy in a large child bearing population. PloS One. 2016; 11 (8): e0161045.

75. Shubina J, Trofimov DY, Barkov IY, Stupko OK, Goltsov AY, Mukosey IS, et al. In silico size selection is effective in reducing false positive NIPS cases of monosomy $X$ that are due to maternal mosaic monosomy X. Prenatal diagnosis. 2017; 37 (13): 1305-10.

76. Bianchi DW, Chudova D, Sehnert AJ, Bhatt S, Murray K, Prosen TL, et al. Noninvasive prenatal testing and incidental detection of occult maternal malignancies. Jama. 2015; 314 (2): 162-9.

77. Ferguson-Smith MA. Placental mRNA in maternal plasma: prospects for fetal screening. Proceedings of the National Academy of Sciences. 2003; 100 (8): 4360-2.

78. Chiu RW, Cantor CR, Lo YD. Non-invasive prenatal diagnosis by single molecule counting technologies. Trends in genetics. 2009; 25 (7): 324-31.

79. ACOG Practice Bulletin № 77: screening for fetal chromosomal abnormalities. Obstet Gynecol. 2007; (109): 217-27.

80. Benn P, Borrell A, Chiu RW, Cuckle H, Dugoff L, Faas B, et al. Position statement from the Chromosome Abnormality Screening Committee on behalf of the Board of the International Society for Prenatal Diagnosis. Prenatal diagnosis. 2015; 35 (8): 725-34.

81. Кащеева Т. К., Кузнецова Т. В., Баранов В. С. Новые технологии и тенденции развития пренатальной диагностики. Журнал акушерства и женских болезней. 2017; 66 (2): 33-39.

82. UK National Screening Committee. www.gov.uk URL. Available from: https://www.gov.uk/government/groups/uk-national-screeningcommittee-uk-nsc (дата обращения: 20.07.2018).

83. Screening in the UK: making effective recommendations 2015 to 2016. Public Health England hosts the UK National Screening Committee URL. Available from: https://assets. publishing.service.gov.uk/government/uploads/system/uploads/ attachment_data/file/538524/Screening in the UK making effective_recommendations_2015_to_2016_180716_final.pdf (дата обращения: 20.07.2018).

84. Analysis of foetal DNA in the woman's blood: non-invasive prenatal testing (NIPT) for trisomy 13, 18 and 21. SFOG Guidelines URL: http://www.nfog.org/files/guidelines/NIPT\%202016\%2006\%20 05\%20.pdf (дата обращения: 20.07.2018).

85. Trisomie 21 : la HAS actualise ses recommandations concernant le dépistage prénatal de la trisomie 21. www.has-sante.fr URL. Available from: https://www.has-sante.fr/portail/jcms/c_2768535/fr/ trisomie-21-la-has-actualise-ses-recommandations-concernant-ledepistage-prenatal-de-la-trisomie-21 (дата обращения: 20.07.2018).

86. Allyse M, Wick MJ. What do the new American College of Medical Genetics and Genomics (ACMG) guidelines mean for the provision of non-invasive prenatal genetic screening? Journal of Obstetrics and Gynaecology. 2017; 37 (6): 795-8.

87. American College of Obstetricians and Gynecologists: Screening for fetal aneuploidy. Obstet Gynecol. 2016; 127 (5): e123-137.

88. Prenatal cell-free DNA screening. National Society of Genetic Counselors. Available from: http://www.nsgc.org/p/bl/et/ blogaid=805\#.WCTELTNR ftw.linkedin.

89. Gregg AR, Skotko BG, Benkendorf JL, Monaghan KG, Bajaj K, Best RG, et al. Noninvasive prenatal screening for fetal aneuploidy, 2016 update: a position statement of the American College of Medical Genetics and Genomics. Genetics in medicine. 2016; 18 (10): 1056-65.

90. Информационно-методическое письмо Минздрава РФ от 19.03.2015 № 15-4/607. Доступно по ссылке: http://www. consultant.ru/document/cons_doc_LAW_177689/.

91. Сухих Г. Т., Трофимов Д. Ю, Барков И. Ю., Донников А. Е., Шубина Е. С., Коростин Д. О. и др. Неинвазивный пренатальный ДНК-скрининг анеуплоидий плода по крови матери методом высокопроизводительного секвенирования. Клинические рекомендации. Акушерство и гинекология. 2016; (6): 3-22.

\section{References}

1. Hassold $T$, Hall H, Hunt P. The origin of human aneuploidy: where we have been, where we are going. Human molecular genetics. 2007; 16 (2): 203-8.

2. Driscoll DA, Gross S. Prenatal screening for aneuploidy. New England Journal of Medicine. 2009; 360 (24): 2556-62.

3. Nagaoka SI, Hassold TJ, Hunt PA. Human aneuploidy: mechanisms and new insights into an age-old problem. Nature Reviews Genetics. 2012; 13 (7): 493-504.

4. Ehrich M, Deciu C, Zwiefelhofer T, Tynan JA, Cagasan L, Tim R, et al. Noninvasive detection of fetal trisomy 21 by sequencing of DNA in maternal blood: a study in a clinical setting. American journal of obstetrics and gynecology. 2011; 204 (3): 205-e1.

5. Morris JK, Mutton DE, Alberman E. Revised estimates of the maternal age specific live birth prevalence of Down's syndrome. Journal of medical screening. 2002; 9 (1): 2-6.

6. Buckley F, Buckley S. Wrongful deaths and rightful lives-screening for Down syndrome. Down Syndrome Research and Practice. 2008; 12 (2): 79-86.

7. Wald NJ, Hackshaw AK. Combining ultrasound and biochemistry in first-trimester screening for Down's syndrome. Prenatal diagnosis. 1997; 17 (9): 821-9.

8. Sillence KA, Madgett TE, Roberts LA, Overton TG, Avent ND. 
Non-invasive screening tools for Down's syndrome: a review. Diagnostics. 2013; 3 (2): 291-314.

9. Choy KW, Kwok YK, Cheng YKY, Wong KM, Wong HK, Leung KO, et al. Diagnostic accuracy of the BACs-on-Beads ${ }^{\mathrm{TM}}$ assay versus karyotyping for prenatal detection of chromosomal abnormalities: a retrospective consecutive case series. BJOG: An International Journal of Obstetrics \& Gynaecology. 2014; 121 (10): 1245-52.

10. Lo YD, Corbetta N, Chamberlain PF, Rai V, Sargent IL, Redman CW, et al. Presence of fetal DNA in maternal plasma and serum. The lancet. 1997; 350 (9076): 485-7.

11. Lo YD, Hjelm NM, Fidler C, Sargent IL, Murphy MF, Chamberlain PF et al. Prenatal diagnosis of fetal RhD status by molecular analysis of maternal plasma. New England Journal of Medicine. 1998; 339 (24): 1734-8.

12. Farina A, Caramelli E, Concu M, Sekizawa A, Ruggeri R, Bovicelli L, et al. Testing normality of fetal DNA concentration in maternal plasma at 10-12 completed weeks' gestation: a preliminary approach to a new marker for genetic screening. Prenatal Diagnosis: Published in Affiliation With the International Society for Prenatal Diagnosis. 2002; 22 (2): 148-52.

13. Bischoff FZ, Lewis DE, Simpson JL. Cell-free fetal DNA in maternal blood: kinetics, source and structure. Human reproduction update. 2005; 11 (1): 59-67.

14. Wang E, Batey A, Struble C, Musci T, Song K, Oliphant A. Gestational age and maternal weight effects on fetal cell-free DNA in maternal plasma. Prenatal diagnosis. 2013; 33 (7): 662-6.

15. Curnow KJ, Gross SJ, Hall MP, Stosic M, Demko Z, Zimmermann B, et al. Clinical experience and follow-up with large scale singlenucleotide polymorphism-based noninvasive prenatal aneuploidy testing. American journal of obstetrics and gynecology. 2014; 211 (5): 527-e1.

16. Tjoa ML, Cindrova-Davies T, Spasic-Boskovic O, Bianchi DW, Burton GJ. Trophoblastic oxidative stress and the release of cellfree feto-placental DNA. The American journal of pathology. 2006; 169 (2): 400-4

17. Alberry M, Maddocks D, Jones M, Abdel Hadi M, Abdel-Fattah S, Avent N, Soothill PW. Free fetal DNA in maternal plasma in anembryonic pregnancies: confirmation that the origin is the trophoblast. Prenatal Diagnosis: Published in Affiliation With the International Society for Prenatal Diagnosis. 2007; 27 (5): 415-8.

18. Gardner RM, Sutherland GR, Shaffer LG. Chromosome abnormalities and genetic counseling. Oxford University Press USA. 2011; (61).

19. Lo YD, Chan KA, Sun H, Chen EZ, Jiang P, Lun FM, et al. Maternal plasma DNA sequencing reveals the genome-wide genetic and mutational profile of the fetus. Science translational medicine. 2010; 2 (61): 61ra91-61ra91.

20. Ivanov M, Baranova A, Butler T, Spellman P, Mileyko V. Nonrandom fragmentation patterns in circulating cell-free DNA reflect epigenetic regulation. BMC genomics. 2015; 16 (13): S1.

21. Sancho M, Diani E, Beato M, Jordan A. Depletion of human histone $\mathrm{H} 1$ variants uncovers specific roles in gene expression and cell growth. PLoS genetics. 2008; 4 (10): e1000227.

22. Jiang P, Lo YMD. The long and short of circulating cell-free DNA and the ins and outs of molecular diagnostics. Trends in Genetics. 2016; 32 (6): 360-71.

23. Geiman TM, Muegge K. DNA methylation in early development. Molecular Reproduction and Development: Incorporating Gamete Research. 2010; 77 (2): 105-13.

24. Sun $\mathrm{K}$, et al. Plasma DNA tissue mapping by genome-wide methylation sequencing for noninvasive prenatal, cancer, and transplantation assessments. Proceedings of the National Academy of Sciences. 2015; 112 (40): E5503-E5512.

25. Sun $\mathrm{K}$, Jiang $\mathrm{P}$, Wong $\mathrm{Al}$, Cheng $\mathrm{YK}$, Cheng $\mathrm{SH}$, Zhang $\mathrm{H}$, et al. Size-tagged preferred ends in maternal plasma DNA shed light on the production mechanism and show utility in noninvasive prenatal testing. Proceedings of the National Academy of Sciences. 2018 115 (22): E5106-E5114.

26. Tamminga S, van Maarle M, Henneman L, Oudejans CB, Cornel MC Sistermans EA. Maternal plasma DNA and RNA sequencing for prenatal testing. Advances in clinical chemistry. 2016; (74): 63-102.

27. Ashoor G, Poon L, Syngelaki A, Mosimann B, Nicolaides $\mathrm{KH}$. Fetal fraction in maternal plasma cell-free DNA at 11-13 weeks gestation: effect of maternal and fetal factors. Fetal diagnosis and therapy. 2012; 31 (4): 237-43.

28. Canick JA, et al. The impact of maternal plasma DNA fetal fraction on next generation sequencing tests for common fetal aneuploidies. Prenatal diagnosis. 2013; 33 (7): 667-74.

29. Wright D, Syngelaki A, Bradbury I, Akolekar R, Nicolaides KH. First-trimester screening for trisomies 21, 18 and 13 by ultrasound and biochemical testing. Fetal diagnosis and therapy. 2014; 35 (2): 118-26.

30. Kim SK, Hannum G, Geis J, Tynan J, Hogg G, Zhao C, et al. Determination of fetal DNA fraction from the plasma of pregnant women using sequence read counts. Prenatal diagnosis. 2015; 35 (8): 810-15

31. Jiang P, Peng $X$, Su $X$, Sun K, Stephanie CY, Chu WI, et al. FetalQuant SD: accurate quantification of fetal DNA fraction by shallow-depth sequencing of maternal plasma DNA. NPJ genomic medicine. 2016; (1): 16013.

32. Peng $X L$, Jiang P. Bioinformatics approaches for fetal DNA fraction estimation in noninvasive prenatal testing. International journal of molecular sciences. 2017; 18 (2): 453.

33. Zimmermann B, Hill M, Gemelos G, Demko Z, Banjevic M, Baner J, et al. Noninvasive prenatal aneuploidy testing of chromosomes $13,18,21, X$, and $Y$, using targeted sequencing of polymorphic loci. Prenatal diagnosis. 2012; 32 (13): 1233-41.

34. Lun FM, Chiu RW, Sun K, Leung TY, Jiang P, Chan KA, et al. Noninvasive prenatal methylomic analysis by genomewide bisulfite sequencing of maternal plasma DNA. Clinical chemistry. DOI: 10.1373/clinchem.2013.212274.

35. Yu SC, et al. Size-based molecular diagnostics using plasma DNA for noninvasive prenatal testing. Proceedings of the National Academy of Sciences. 2014; 111 (23): 8583-8.

36. Cirigliano $V$, Ordoñez $E$, Rueda $L$, Syngelaki A, Nicolaides $K H$. Performance of the neoBona test: a new paired-end massively parallel shotgun sequencing approach for cell-free DNA-based aneuploidy screening. Ultrasound in Obstetrics \& Gynecology. 2017; 49 (4): 460-4.

37. Straver R, Oudejans C, Sistermans EA, Reinders MJ. Calculating the fetal fraction for noninvasive prenatal testing based on genome-wide nucleosome profiles. Prenatal diagnosis. 2016; 36 (7): 614-21.

38. Kim SK, Hannum G, Geis J, Tynan J, Hogg G, Zhao C, Boom D. Determination of fetal DNA fraction from the plasma of pregnant women using sequence read counts. Prenatal diagnosis. 2015; 35 (8): 810-5

39. Chen S, Lau TK, Zhang C, Xu C, Xu Z, Hu P, et al. A method for noninvasive detection of fetal large deletions/duplications by low coverage massively parallel sequencing. Prenatal diagnosis. 2013; 33 (6): 584-90.

40. Van den Veyver IB. Recent advances in prenatal genetic screening and testing. F1000 Research. 2016; 5 (F1000 Faculty Rev): 2591.

41. Peters D, Chu T, Yatsenko SA, Hendrix N, Hogge WA, Surti U, et al. Noninvasive prenatal diagnosis of a fetal microdeletion syndrome. New England Journal of Medicine. 2011; 365 (19): 1847-8.

42. Jensen TJ, Dzakula Z, Deciu C, van den Boom D, Ehrich M. Detection of microdeletion 22q11. 2 in a fetus by next-generation sequencing of maternal plasma. Clinical chemistry. 2012; 58 (7): 1148-51.

43. Srinivasan A, Bianchi DW, Huang $H$, Sehnert AJ, Rava RP. Noninvasive detection of fetal subchromosome abnormalities via deep sequencing of maternal plasma. The American Journal of Human Genetics. 2013; 92 (2): 167-76.

44. Lefkowitz RB, Tynan JA, Liu T, Wu Y, Mazloom AR, Almasri E, et al. Clinical validation of a noninvasive prenatal test for genomewide detection of fetal copy number variants. American journal of obstetrics and gynecology. 2016; 215 (2): 227-e1.

45. Straver R, Sistermans EA, Holstege H, Visser A, Oudejans CB, Reinders MJ. WISECONDOR: detection of fetal aberrations from shallow sequencing maternal plasma based on a within-sample comparison scheme. Nucleic acids research. 2013; 42 (5): e31-e31.

46. Zhao C, Tynan J, Ehrich M, Hannum G, McCullough R, Saldivar JS, Deciu C. Detection of fetal subchromosomal abnormalities by sequencing circulating cell-free DNA from maternal plasma. Clinical chemistry. 2015. 
47. Nicolaides KH, Syngelaki A, Gil M, Atanasova V, Markova D. Validation of targeted sequencing of single-nucleotide polymorphisms for non-invasive prenatal detection of aneuploidy of chromosomes 13, 18, 21, X, and Y. Prenatal diagnosis. 2013; 33 (6): 575-9.

48. Wapner RJ, Babiarz JE, Levy B, Stosic M, Zimmermann B, Sigurjonsson S, Hu J. Expanding the scope of noninvasive prenatal testing: detection of fetal microdeletion syndromes. American journal of obstetrics and gynecology. 2015; 212 (3): 332-e1.

49. Costa JM, Benachi A, Gautier E. New strategy for prenatal diagnosis of X-linked disorders. New England Journal of Medicine. 2002; 346 (19): 1502.

50. Lo YD, Hjelm NM, Fidler C, Sargent IL, Murphy MF, Chamberlain PF, et al. Prenatal diagnosis of fetal RhD status by molecular analysis of maternal plasma. New England Journal of Medicine. 1998; 339 (24): 1734-8.

51. Tang NL, Leung TN, Zhang J, Lau TK, Lo YD. Detection of fetalderived paternally inherited X-chromosome polymorphisms in maternal plasma. Clinical chemistry. 1999; 45 (11): 2033-5.

52. Bustamante-Aragonés A, de Alba MR, Perlado S, Trujillo-Tiebas MJ, Arranz JP, Díaz-Recasens J, et al. Non-invasive prenatal diagnosis of single-gene disorders from maternal blood. Gene. 2012; 504 (1): 144-9.

53. Amicucci P, Gennarelli M, Novelli G, Dallapiccola B. Prenatal diagnosis of myotonic dystrophy using fetal DNA obtained from maternal plasma. Clinical chemistry. 2000; 46 (2): 301-2.

54. Vermeulen C, Geeven G, de Wit E, Verstegen MJ, Jansen RP, van Kranenburg $M$, et al. Sensitive monogenic noninvasive prenatal diagnosis by targeted haplotyping. The American Journal of Human Genetics. 2017; 101 (3): 326-39.

55. Tong YK, Jin S, Chiu RW, Ding C, Chan KA, Leung TY, et al. Noninvasive prenatal detection of trisomy 21 by an epigeneticgenetic chromosome-dosage approach. Clinical chemistry. 2010; 56 (1): 90-8.

56. Tsui DW, Lam YD, Lee WS, Leung TY, Lau TK, Lau ET, et al. Systematic identification of placental epigenetic signatures for the noninvasive prenatal detection of Edwards syndrome. PloS one. 2010; 5 (11): e15069.

57. Yuen RK, Penaherrera MS, Von Dadelszen P, McFadden DE, Robinson WP. DNA methylation profiling of human placentas reveals promoter hypomethylation of multiple genes in early-onset preeclampsia. European Journal of Human Genetics. 2010; 18 (9): 1006.

58. Blair JD, Yuen RK, Lim BK, McFadden DE, von Dadelszen P, Robinson WP. Widespread DNA hypomethylation at gene enhancer regions in placentas associated with early-onset preeclampsia. Molecular human reproduction. 2013; 19 (10): 697-708.

59. Chu T, Bunce K, Shaw P, Shridhar V, Althouse A, Hubel C, et al. Comprehensive analysis of preeclampsia-associated DNA methylation in the placenta. PLoS One. 2014; 9 (9): e107318.

60. Hahn S, Rusterholz C, Hösli I, Lapaire O. Cell-free nucleic acids as potential markers for preeclampsia. Placenta. 2011; (32): S17-S20.

61. Bianchi DW, Parker RL, Wentworth J, Madankumar R, Saffer C Das AF, et al. DNA sequencing versus standard prenatal aneuploidy screening. New England journal of medicine. 2014; 370 (9): 799-808.

62. Norton ME, Jacobsson B, Swamy GK, Laurent LC, Ranzini AC, Brar $\mathrm{H}$, et al. Cell-free DNA analysis for noninvasive examination of trisomy. New England Journal of Medicine. 2015; 372 (17): 1589-97.

63. Wataganara T, Peter I, Messerlian GM, Borgatta L, Bianchi DW. Inverse correlation between maternal weight and second trimester circulating cell-free fetal DNA levels. Obstetrics \& Gynecology. 2004; 104 (3): 545-50.

64. Rumyantsev AG, Kurcer MA, Mareeva JM, Misjurin AV, Roumiantsev SA, Ustjugov AJ. Clinical significance of the fetal microchimerism for mother. Cellular Transplantation \& Tissue Engineering. 2012; 7 (2): 103-111.

65. Wang $Y$, et al. Maternal mosaicism is a significant contributor to discordant sex chromosomal aneuploidies associated with noninvasive prenatal testing. Clinical chemistry. 2014; 60 (1): 251-9.

66. Bianchi DW. Cherchez la femme: maternal incidental findings can explain discordant prenatal cell-free DNA sequencing results. Genetics in Medicine. 2017; DOI: 10.1038/gim.2017.219.

67. Hartwig TS, Ambye L, Sørensen S, Jørgensen FS. Discordant non-invasive prenatal testing (NIPT)-a systematic review. Prenatal diagnosis. 2017; 37 (6): 527-39

68. Attilakos G, Maddocks DG, Davies T, Hunt LP, Avent ND, Soothill PW, et al. Quantification of free fetal DNA in multiple pregnancies and relationship with chorionicity. Prenatal diagnosis. 2011; 31 (10): 967-72.

69. Bevilacqua E, Gil MM, Nicolaides KH, Ordoñez E, Cirigliano V, Dierickx $\mathrm{H}$, et al. Performance of screening for aneuploidies by cell-free DNA analysis of maternal blood in twin pregnancies. Ultrasound in Obstetrics \& Gynecology. 2015; 45 (1): 61-6.

70. Curnow KJ, Wilkins-Haug L, Ryan A, Kırkızlar E, Stosic M, Hall MP, et al. Detection of triploid, molar, and vanishing twin pregnancies by a single-nucleotide polymorphism-based noninvasive prenatal test. American journal of obstetrics and gynecology. 2015; 212 (1): 79-e1

71. Gil MM, Quezada MS, Revello R, Akolekar R, Nicolaides KH. Analysis of cell-free DNA in maternal blood in screening for fetal aneuploidies: updated meta-analysis. Ultrasound in obstetrics \& gynecology. 2015; 45 (3): 249-66.

72. Zhang $H$, Gao $Y$, Jiang F, Fu M, Yuan $Y$, Guo $Y$, et al. Noninvasive prenatal testing for trisomies 21, 18 and 13: clinical experience from 146958 pregnancies. Ultrasound in Obstetrics \& Gynecology. 2015; 45 (5): 530-8.

73. Russell LM, Strike P, Browne CE, Jacobs PA. X chromosome loss and ageing. Cytogenetic and genome research. 2007; 116 (3): 181-5.

74. Samango-Sprouse C, Kırkızlar E, Hall MP, Lawson P, Demko Z, Zneimer SM, et al. Incidence of $X$ and $Y$ chromosomal aneuploidy in a large child bearing population. PloS One. 2016; 11 (8): e0161045.

75. Shubina J, Trofimov DY, Barkov IY, Stupko OK, Goltsov AY Mukosey IS, et al. In silico size selection is effective in reducing false positive NIPS cases of monosomy $X$ that are due to maternal mosaic monosomy X. Prenatal diagnosis. 2017; 37 (13): 1305-10.

76. Bianchi DW, Chudova D, Sehnert AJ, Bhatt S, Murray K, Prosen TL, et al. Noninvasive prenatal testing and incidental detection of occult maternal malignancies. Jama. 2015; 314 (2): 162-9.

77. Ferguson-Smith MA. Placental mRNA in maternal plasma: prospects for fetal screening. Proceedings of the National Academy of Sciences. 2003; 100 (8): 4360-2.

78. Chiu RW, Cantor CR, Lo YD. Non-invasive prenatal diagnosis by single molecule counting technologies. Trends in genetics. 2009; 25 (7): 324-31.

79. ACOG Practice Bulletin \# 77: screening for fetal chromosomal abnormalities. Obstet Gynecol. 2007; (109): 217-27.

80. Benn P, Borrell A, Chiu RW, Cuckle H, Dugoff L, Faas B, et al. Position statement from the Chromosome Abnormality Screening Committee on behalf of the Board of the International Society for Prenatal Diagnosis. Prenatal diagnosis. 2015; 35 (8): 725-34.

81. Kascheeva TK, Kuznetzova TV, Baranov VS. New technologies and trends of prenatal diagnostics. Journal of obstetrics and woman disease. 2017; 66 (2): 33-39.

82. UK National Screening Committee. www.gov.uk URL. Available from: https://www.gov.uk/government/groups/uk-national-screeningcommittee-uk-nsc (data obrashhenija: 20.07.2018).

83. Screening in the UK: making effective recommendations 2015 to 2016. Public Health England hosts the UK National Screening Committee URL. Available from: https://assets. publishing.service.gov.uk/government/uploads/system/uploads/ attachment_data/file/538524/Screening_in_the_UK__making_ effective_recommendations_2015_to_2016_180716_final.pdf (data obrashhenija: 20.07.2018)

84. Analysis of foetal DNA in the woman's blood: non-invasive prenatal testing (NIPT) for trisomy 13, 18 and 21. SFOG Guidelines URL. Available from: http://www.nfog.org/files/guidelines/NIPT\%20 2016\%2006\%2005\%20.pdf (data obrashhenija: 20.07.2018).

85. Trisomie 21 : la HAS actualise ses recommandations concernant le dépistage prénatal de la trisomie 21. www.has-sante.fr URL. Available from: https://www.has-sante.fr/portail/jcms/c_2768535/fr/ trisomie-21-la-has-actualise-ses-recommandations-concernant-le- 
depistage-prenatal-de-la-trisomie-21 (data obrashhenija: 20.07.2018)

86. Allyse M, Wick MJ. What do the new American College of Medical Genetics and Genomics (ACMG) guidelines mean for the provision of non-invasive prenatal genetic screening? Journal of Obstetrics and Gynaecology. 2017; 37 (6): 795-8.

87. American College of Obstetricians and Gynecologists: Screening for fetal aneuploidy. Obstet Gynecol. 2016; 127 (5): e123-137.

88. Prenatal cell-free DNA screening. National Society of Genetic Counselors. Available from: http://www.nsgc.org/p/bl/et/ blogaid=805\#.WCTELTNRftw.linkedin

89. Gregg AR, Skotko BG, Benkendorf JL, Monaghan KG, Bajaj K, Best RG, et al. Noninvasive prenatal screening for fetal aneuploidy,
2016 update: a position statement of the American College of Medical Genetics and Genomics. Genetics in medicine. 2016; 18 (10): 1056-65.

90. Informacionno-metodicheskoe pis'mo Minzdrava RF ot 19.03.2015 \# 15-4/607. Available from: http://www.consultant. ru/document/cons_doc_LAW_177689/.

91. Sukhikh GT, Trofimov DYu, Barkov IYu, Donnikov AE, Shubina ES, Korostin DO, et al. Non-invasive prenatal DNA-screening of fetus aneuploidies using maternal blood based on high-throuhgput sequencing. Clinical recommendations. Obstetrics and gynecology. 2016; (6): 3-22. 